\title{
Mechanism of Dissociation Kinetics in Polyelectrolyte Complex Micelles
}

\author{
Hao Wu, Jeffrey M. Ting, ${ }^{\dagger, \ddagger}$ and Matthew V. Tirrell ${ }^{*,+, \dagger}$ \\ $\dagger$ Pritzker School of Molecular Engineering, The University of Chicago, Chicago, IL 60637, \\ United States \\ $\ddagger$ Argonne National Laboratory, Lemont, IL 60439, United States \\ E-mail: mtirrell@uchicago.edu \\ Phone: +1 7738342001
}

\begin{abstract}
Polyelectrolyte-based nanoscale self-assemblies, such as micelles, possess diverse desirable attributes such as capability for sequestering and protecting biomacromolecules against inhospitable environments, responsiveness to external stimuli, and tunability of physical behavior. However, little is known on the mechanisms of dissociation when micelles encounter and respond to environmental changes. Using salt-jump, timedependent, light scattering, the pathway of dissociation is observed in polyelectrolyte complex micelles that have complex cores and neutral coronas. The micelle dissociation kinetics appear to be a three-staged process, in good agreement with the scattering data. Using kinetic models of amphiphilic block copolymer micelles in polyelectrolyte complexation-driven micelles, we derive an analytical expression for dissociation relaxation rates as a function of solvent temperature, salt concentration, and the length of the charged polymer blocks. The theoretical predictions are compatible with the experimental data from light scattering experiments. This study demonstrates experimentally the relaxation kinetics of polyelectrolyte complex micelle dissociation and
\end{abstract}


illustrates the underlying mechanism governing the dissociation kinetics. It is anticipated that these findings can be generalized to other electrostatic interaction-driven self-assemblies to better understand the relationship among the kinetics of dissociation, constituent polymer properties, and environmental parameters.

\section{INTRODUCTION}

Ionic complexes formed by charged macromolecular units in natural systems play an important role in membraneless organelle formation, ${ }^{1}$ protein folding, ${ }^{2-4}$ and nucleotide transportation. ${ }^{5-7}$ Synthetic polymers bearing opposite charged moieties also form polyelectrolyte complexes via associative phase separation. ${ }^{8-15}$ These ionic complexation-driven assemblies are of longstanding interest because of their broad industrial applications including underwater adhesives, ${ }^{16}$ encapsulants, ${ }^{17,18}$ delivery, ${ }^{19,20}$ and smart hydrogels. ${ }^{21-23}$

Polyelectrolyte complex (PEC) micelles are a type of nanoscale self-assembly that possesses micellar cores of ionic complexes and coronas of neutral polymers. Polyelectrolyte complex micelles perform well in gene and protein delivery since positively-charged polymers can condense large nucleic acids into small nanostructures and neutralize the negatively-charged moieties on the nucleic acid chains, protecting them from potential enzymatic degradation and promoting successful transfection into various cell types. ${ }^{24-26}$ Being non-covalent assemblies, they can dissociate to deliver the payload.

However, despite its significance, knowledge of the underlying mechanism that governs PEC micelle dissociation kinetics remains limited. The vast majority of the efforts of the past two decades have focused on the static properties of PEC micelles, such as their stimuliresponsiveness, co-micellization with various biological macromolecules, and therapeutic applications. ${ }^{27}$

While direct studies of the dissociation (or formation) kinetics in polyelectrolyte complex micelles have been reported rarely to date, previous efforts and progress on micelles formed by low weight surfactants and nonionic block copolymers may provide initial insights. In the 
1970s, Aniansson and Wall proposed two possible mechanisms for the kinetics of dissociation in surfactant micelles: (1) a step-wise single chain expulsion and insertion mechanism and (2) a collective micelle fission and fusion mechanism. ${ }^{28}$ Later, experimental observations using light scattering techniques clearly confirmed that the kinetics of surfactant micelle dissociation-formation equilibrium can be characterized by two well-separated relaxation processes. Based on that, Halperin and Alexander developed a similar theory for amphiphilic block copolymer (ABC) micelles. ${ }^{29}$ They claimed that micelle fission (or fusion) may be deactivated due to the high free energy penalty it incurs owing to the coronal interaction, which scales as

$$
U_{\text {fiss }} \simeq N_{B}^{2 / 3}\left(P_{1} / P^{2}\right)
$$

where $N_{B}$ is the length of the core-forming block, and $P_{1}$ and $P$ are the aggregation number of the fissionable aggregate and the initial micelle, respectively. The fission activation energy clearly minimizes when $P_{1}=1$, which favors the single chain expulsion mechanism. It is noteworthy that this result is only valid for systems that undergo small deviations from equilibrium states. Results from a few experiments suggested that the single chain expulsion/insertion alone can not explain the phenomena well. For example, Esselink et al. investigated the evolution of mixed $\mathrm{ABC}$ micelles formed by two polymers that have the same composition but different coronal block lengths, and pointed out that the redistribution of polymer chains among micelles proceeds mainly via micelle fusion. ${ }^{30}$ Dormidontova predicted theoretically that micelle fission is a slow process at dynamic equilibrium states but plays a major role when micelles re-equilibrate from a large perturbation (such as during temperature-jump experiments). ${ }^{31}$ Compared to unimer expulsion where the entropic penalty comes from the exposure of the solvophobic chains in selective solvent, micelle fission needs to overcome the free energy increase corresponding to the separation of micellar cores. The fission activation energy, $U_{f i s s}$, is given as:

$$
U_{\mathrm{fiss}} \simeq P_{\mathrm{eq}}^{5 / 6}\left[P_{1}^{2 / 3}+P_{2}^{2 / 3}-\left(P_{1}+P_{2}\right)^{2 / 3}\right]
$$


where $P_{e q}, P_{1}$, and $P_{2}$ represent the aggregation numbers of the pre-fission micelles in equilibrium and post-fission micelle 1 and 2, respectively. It predicts that when a micelle system is far away from its equilibrium state, micelle fission or fusion becomes dominant. Recently, experimental efforts have been made to observe micelle fission directly. For example, Burke et al. observed that fragmentation of block copolymer micelles happened when micelles undergo morphological transitions from spherical micelles to rod-like aggregates; ${ }^{32}$ Rharbi reported that, even at equilibrium, micelle fission took place in PEO-PPO-PEO micelles, but with a rate $10^{6}$ slower than that of chain expulsion and insertion. ${ }^{33}$

In comparison, experimental work on the dissociation kinetics of PEC micelles is scarce. Despite the stark contrast of the driving forces for self-assembly, PEC micelles have been oftentimes understood in an analogous way to ABC micelles. This comparison may be plausible with respect to static micellar structures, which have been the focus of attention so far. We recently reported that the long-range electrostatic interactions do not significantly affect micelle-micelle correlation, demonstrating that PEC micelles in dilute solutions interact in a similar way to their uncharged counterparts. ${ }^{34}$ However, when it comes to kinetic transitions in micelles, distinctions emerge. First, ABC micelles can often be a single component system that contains only a single type of polymer with core-forming solvophoic block; PEC micelles in most cases are a multicomponent system in which the interplay between positively-charged blocks, negatively-charged blocks, one or two coronal blocks, counterions, and solvent (normally water) complicates micellar dynamics. Second, unlike ABC micelles, the core chains in PEC micelles are hydrophilic, and exist in a complex state via ionic interactions. This means the expulsion of a chain from micelle cores exposes no unfavorable chains in solvent but depending on the ionic environment may need to counteract the Coulomb attraction between charged moieties. The breakup of the electrostatic bonds may retard the kinetics. With a model PEC micelle system, we have studied chain exchange using time-resolved small-angle neutron scattering indicates that these micelles in equilibrium remain frozen up to 40 hours. Third, ABC micelles often have solid-like or glassy cores, whereas PEC micelle cores may 
be fluidic, and contain a large amount of water (ca. $30-90 \%$ ), which is subject to environmental factors such as salt ion concentration and system temperature. All these features combined complicate studies on the mechanism of PEC micelle dissociation or formation. Zhang et al. investigated the salt-induced disassembly kinetics of polyelectrolyte complex micelles formed by poly(ethlyene oxide)- $b$-poly(sodium styrene sulfonate) (PEO-b-PSS) and poly (ethylene oxide)- $b$-poly (quaternized dimethyl amino methacrylate) (PEO-b-PQDMA). They fit the relaxation curves by a double-exponential function, in which the fast relaxation process is attributed to the initial complex formation and the slow relaxation is attributed to a micelle fusion-fission mechanism.

In this article, we aim to make strides toward understanding the mechanism of dissociation kinetics in polyelectrolyte complex-based micelles. By combining knowledge of polyelectrolyte complexation and micelle scaling laws, we (1) develop a theoretical framework to describe the kinetic pathway of PEC micelle dissociation, (2) illustrate the rationale behind it, (3) derive an analytical expression of fission relaxation kinetics as a function of polyelectrolyte molecular weights, salt concentration and temperature, and (4) compare our experimental results with theoretical predictions. The experimental system we employ here consists of a positively charged diblock polyelectrolyte, poly(ethylene oxide)-block-poly(vinyl benzyl trimethylammonium chloride $)\left(\mathrm{PEO}_{225}-b-\mathrm{PVBTMA}_{100}\right)$, and a negatively charged homopolymer, poly(acrylic acid sodium) $\left(\mathrm{PAA}_{158}\right) \cdot{ }^{35,36}$ The subscripts indicate the numbers of repeat units. The PVBTMA block has been demonstrated as an effective cationic vector for DNA encapsulation and delivery, while the PAA block has been extensively studied as a proxy for weakly charged biomacromolecules. ${ }^{37,38}$ The physical properties of the micelles in equilibrium are characterized by a combination of techniques including dynamic light scattering, small-angle X-ray scattering, and cryogenic electron microscopy. The salt-dependent evolution of PEC micelles upon an abrupt salinity ascendance is investigated using time-resolved static light scattering. We have developed a quantitative model to predict the dependence of the dissociation rate on the ionic chain length, temperature and salt concentration, which 
approximately agree with our data and can be further examined.

\section{RESULTS AND DISCUSSIONS}

Characterization of PEC Micelles. We characterized the morphology, size, and internal structure of the PEC micelles by employing a combination of techniques including dynamic light scattering (DLS), small-angle X-ray scattering (SAXS), and cryogenic electron microscopy (cryo-TEM). As the DLS analysis shows in Figure 1B, the PEC micelles exhibit a monomodal size distribution, and the hydrodynamic radius is centered at $30 \mathrm{~nm}$ with a narrow distribution. As seen in Figure 1C, SAXS was further employed to determine the sizes of micelle core and corona. The SAXS profile exhibits a characteristic pattern of spherical objects: at low q values, the curve follows a quasi-plateau and transitions to a intensity drop that scales with $\mathrm{q}$ with a power law of -4 at the middle $\mathrm{q}$ region. At the high

$\mathrm{q}$ region, the intensity decreases along $\mathrm{q}$ with a power law of $-2.3,{ }^{39-41}$ which is characteristic of the scattering from the individual constituent polymers. Using a polydisperse core-shell model, we extracted the radius of the core and the thickness of the corona, which are 10.3 $\mathrm{nm}$ and $6.8 \mathrm{~nm}$, respectively. The morphology of the PEC mielles was further visualized by Cryo-TEM in Figure 1D. Due to the light contrast between the coronas and the background, Cryo images only shows the micelle cores. The cores are generally spheroidal, and the average radius is around $13 \mathrm{~nm}$. Results from DLS, SAXS, and Cryo-images are generally in line with one another.

Kinetics of Salt-Induced Dissociation of PEC Micelles. A typical time-dependent light scattering experiment is illustrated in Scheme 1 in the Experimental Section. The time evolution of light scattering was performed under two scenarios: $(i)$ the temperaturedependence case where a micelle solution containing $1.0 \mathrm{mg} \mathrm{mL} \mathrm{mLO}_{225}-b$-PVBTMA $\mathrm{PE}_{100} / \mathrm{PAA}_{158}$ is measured, upon a salinity jump from 0 to $500 \mathrm{mM} \mathrm{NaCl}$, at three different temperatures, i.e. 20,37 , and $57^{\circ} \mathrm{C}$, respectively, and (ii) the salt-dependence case where a micelle solution 

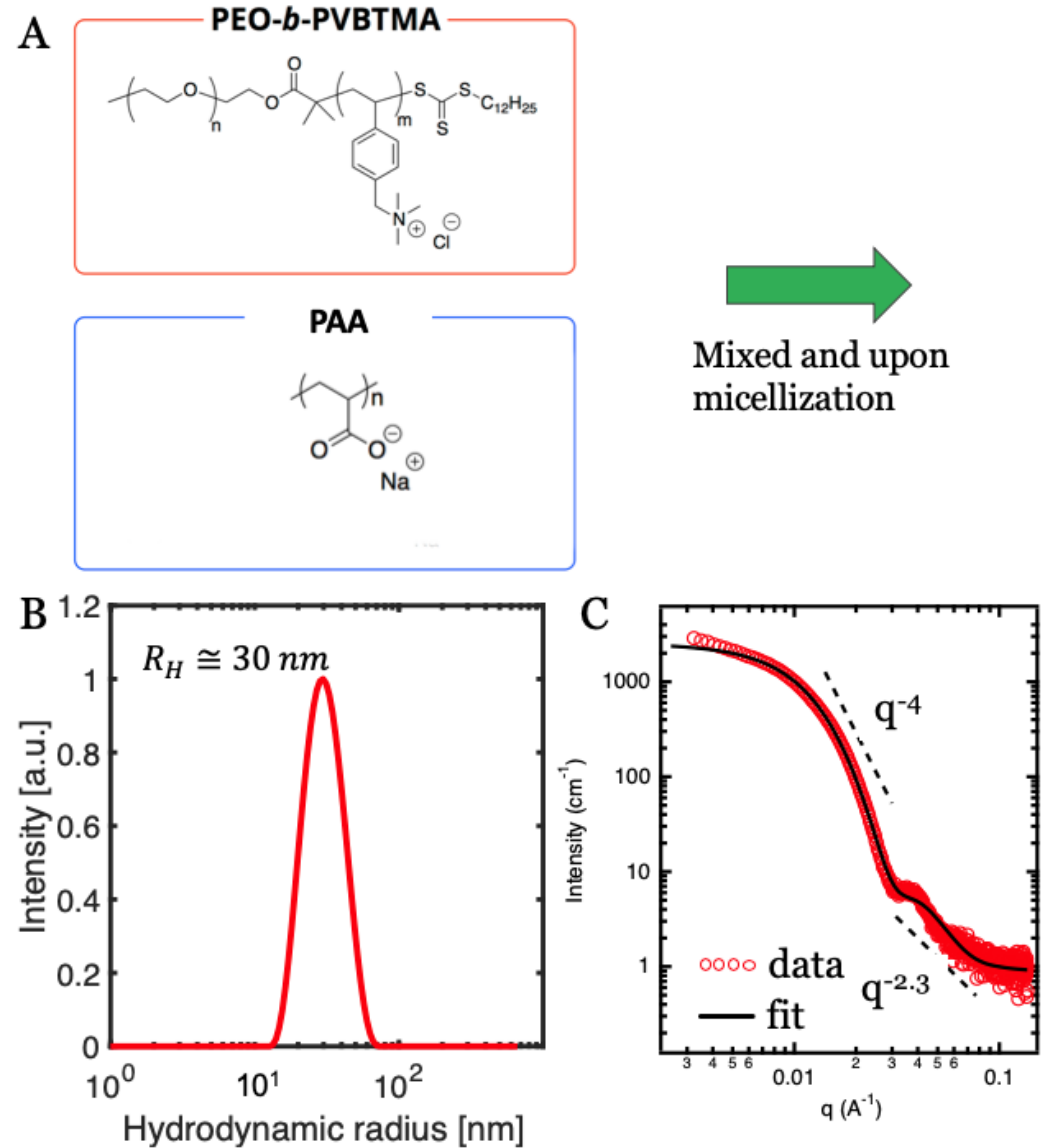

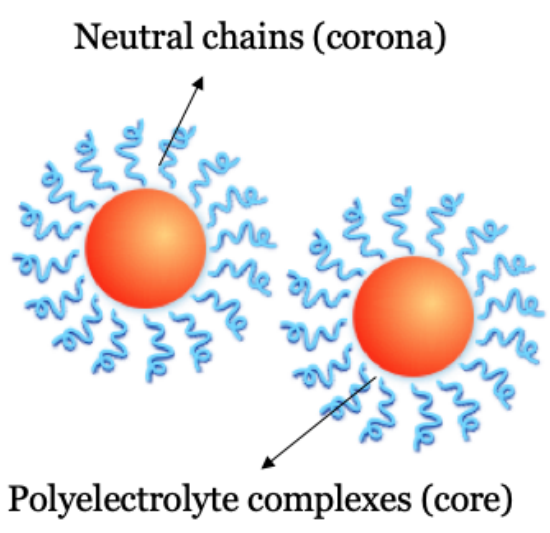

$\mathrm{D}$

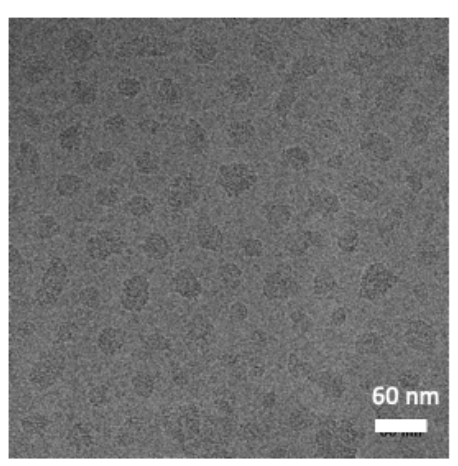

Figure 1: (A) Schematic representation of the core-corona strucure micelles formed upon the micellization of the oppositely charged polymers, i. e. PEO- $b$-PVBTMA and the poly(acrylic acid sodium) (PAA). Chemical structures are shown. (B) The size distribution and apparent hydrodynamic radius determined by DLS analysis using the REPES algorithm. The apparent hydrodynamic radis, $R_{H}$, is about $30 \mathrm{~nm}$. (C) SAXS curve of the PEC micelles and the fitting using a polydisperse core-corona sphere model. (D) Representative cryo-TEM images to visualize the morphology of the PEC micelles. The average size of micellar cores is about $26 \mathrm{~nm}$. The scale bar is $60 \mathrm{~nm}$. 
with $1.0 \mathrm{mg} \mathrm{mL}{ }^{-1} \mathrm{PEO}_{225}$-b-PVBTMA $100 / \mathrm{PAA}_{158}$ was measured when the solvent salt concentration jumps to $300 \mathrm{mM}, 400 \mathrm{mM}, 500 \mathrm{mM}$, and $600 \mathrm{mM}$, respectively. As illustrated by Figure 3 and 3 , the induced disassembly causes strong decrease of the scattering intensities.

We take the structure factor as unity because the interparticle interaction is minimal at such a dilute polymer concentration, and also take the form factor as unity since $q R_{\text {micelle }}<1$, where $R_{\text {micelle }}$ is the radius of the salt-free micelles, $q$ is the scattering wave vector, $\lambda_{0}$ is the incident laser wavelength that equals to $637 \mathrm{~nm}, n$ is the solvent refractive index which is about 1.332 for $\mathrm{H}_{2} \mathrm{O}$ at room temperature, and is the measuring angle which is $90^{\circ}$. Therefore, the scattering intensity is written as ${ }^{42}$

$$
I(t) \approx\left(\frac{d n}{d c}\right)^{2}\left(C-C_{C M C}\right) P_{\text {mean }}(t) M_{w}
$$

where $\frac{d n}{d c}$ is the reflective index increment, $C$ is the weight concentration of the polymers, $C_{C M C}$ is the critical micelle concentration which is primarily a function of the solvent salt concentration, $P_{\text {mean }}(t)$ is the average aggregation number and is time dependent, and $M_{w}$ is the molecular weight of a building block. A building block is defined as a neutralized group containing a diblock polyelectrolyte chain plus the amount of oppositely charged homopolymer needed to neutralize it. In this case, $M_{w}$ approximately equals $150 \mathrm{~kg} \mathrm{~mol}^{-1}$. It is shown, for a given system, that the time evolution of the scattering intensity is only proportional to the average aggregation number. Equation (3) is therefore simplified to be

$$
I(t) \approx P_{\text {mean }}(t)
$$

Trial fits of a single exponential or a sum of two exponential functions do not give satisfactory results, which indicates the kinetic of the dissociation process is inherently more complicated. Similar results have been found and reported by us in kinetically trapped polyelectrolyte complexes. ${ }^{36}$ Satisfactory fits are only obtained when we fit the dissociation of the micelles 
with an Avrami-type model in the form of a compressed exponential function as follows:

$$
P_{\text {mean }}(t) \approx \exp \left[-(t / \tau)^{\beta}\right]
$$

where $\tau$ is the relaxation rate and $\beta$ is the exponential exponent that relates to the type of (de)nucleation and shrinking (or growth) geometry. The Avrami phenomenological model has been used to describe the relaxation kinetics in far-from-equilibrium systems, such as isothermal phase transformations ${ }^{43}$ crystallization kinetics in polymer blends,${ }^{44}$ aging dynamics in colloidal gels, ${ }^{45}$ adsorption kinetics of polyelectrolytes on solution interfaces ${ }^{46}$ and morphological transitions in $\mathrm{ABC}$ micelles. ${ }^{47}$ The exponential exponent, $\beta$, is assumed to range from 1 to 4 and is an indicator of the geometric dimension of the denucleation: Avrami exponent $\beta=1.0$ denotes the one-dimensional rodlike denucleation and $\beta=2.0$ indicates the two-dimensional disklike denucleation. ${ }^{48,49}$ In micelle dynamics, first-order kinetics $(\beta=1.0)$ corresponds to the single chain expulsion/insertion mechanism. ${ }^{29,50}$ Second-order kinetics $(\beta=2.0)$ has also been reported in certain $\mathrm{ABC}$ micelles. Meli et al. reported that the relaxation kinetics in poly(ethylene oxide)- $b$-poly(butadiene) micelles in ionic liquids over a broad range of temperature and concentrations can be well described by an Avrami-type relaxation function with an exponent of $2 .{ }^{51}$ They attributed this temperature-dependence relaxation behavior to a micellar fission/fusion mechanism. Here, because the salinity jump renders micelle systems far way from equilibrium, we attempt to use the Avrami model to elucidate the relaxation behaviors in the PEC micelles.

The fitting results are shown in Table 1. It is observed that $(i)$ the relaxation time decreases as temperature increases or salt concentration increases, (ii) all the exponential exponents are in the range of 1 and 2, and (iii) the exponent of the low temperature is in proximity to 2 but decreases when the temperature increases. The acceleration of the micellar dissociation upon temperature increase is probably due to the thermally activated motion of the polymeric chains as well as the enhanced random forces from the solvent 

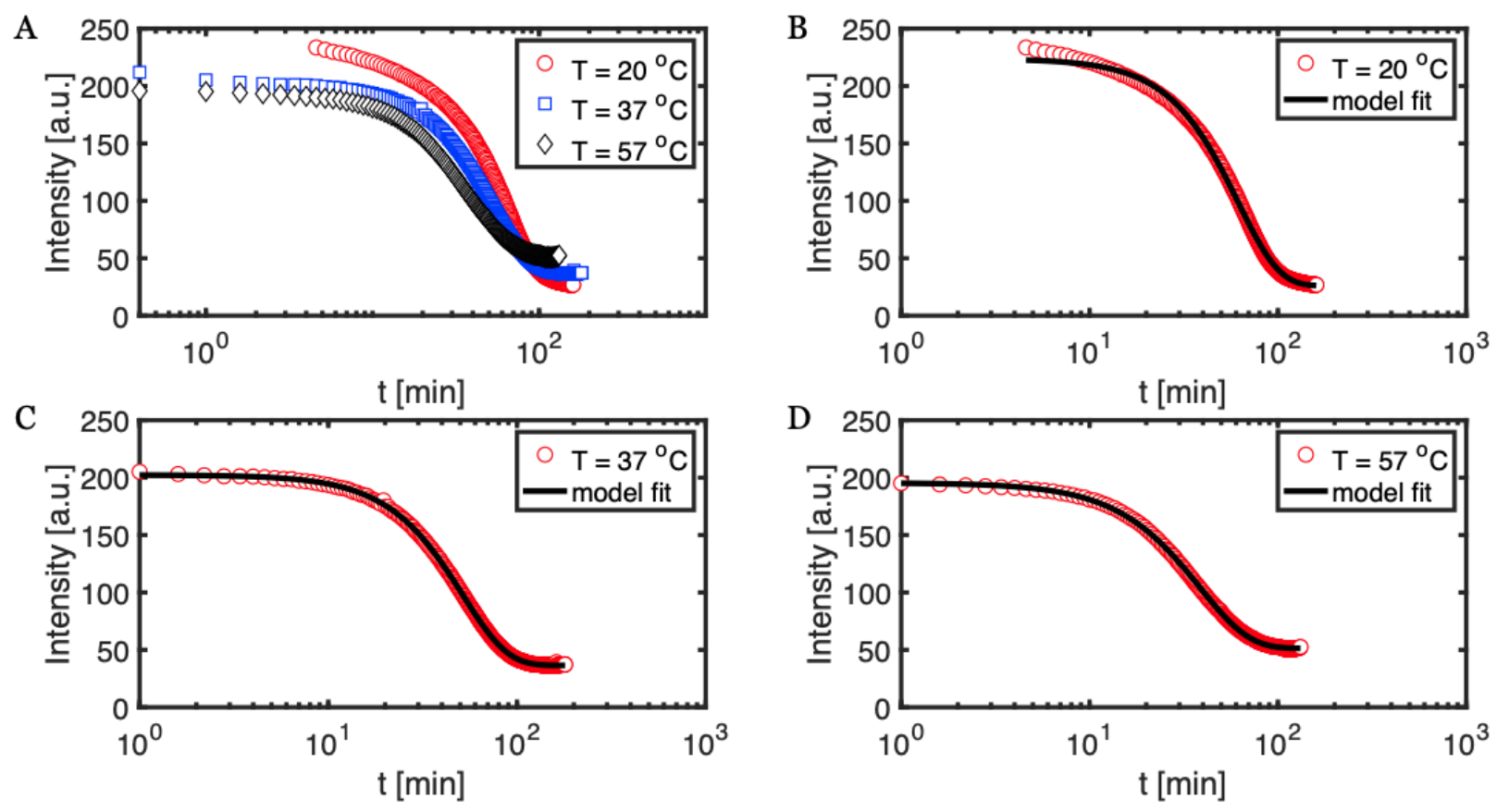

Figure 2: (A) The time evolution of light scattering intensity from three temperatures during the kinetics upon the salinity jumps to $500 \mathrm{mM} \mathrm{NaCl}$ in the $1.0 \mathrm{mg} \mathrm{mL}^{-1} \mathrm{PEO}_{225}-b$ PVBTMA $_{100} / \mathrm{PAA}_{158}$ solution. The Avrami model fits of the temperature-dependence dissociation profiles at (B) $20{ }^{\circ} \mathrm{C}$, (C) $37{ }^{\circ} \mathrm{C}$, and (D) $57{ }^{\circ} \mathrm{C}$, respectively. The red lines are the experimental data and the black lines represent the fits. The relaxation rates from model fits are $61.54,51.77$, and $38.57 \mathrm{~min}$ at $20^{\circ} \mathrm{C}, 37^{\circ} \mathrm{C}$, and $57^{\circ} \mathrm{C}$, respectively.

Table 1: The relaxation rates and exponential exponents of micelle dissociation at different temperatures and various salt concentrations.

\begin{tabular}{cccc}
\hline Temp $(\mathrm{K})$ & $\mathrm{C}_{\mathrm{s}}(\mathrm{mM})$ & $\tau(\min )$ & $\beta$ \\
\hline 293 & & 61.5 & 2.00 \\
310 & 0 & 57.8 & 1.84 \\
330 & & 38.6 & 1.66 \\
\hline \multirow{3}{*}{293} & 300 & 51.9 & 0.82 \\
& 400 & 35.2 & 1.43 \\
& 500 & 27.4 & 2.03 \\
& 600 & 10.9 & 1.94 \\
\hline
\end{tabular}



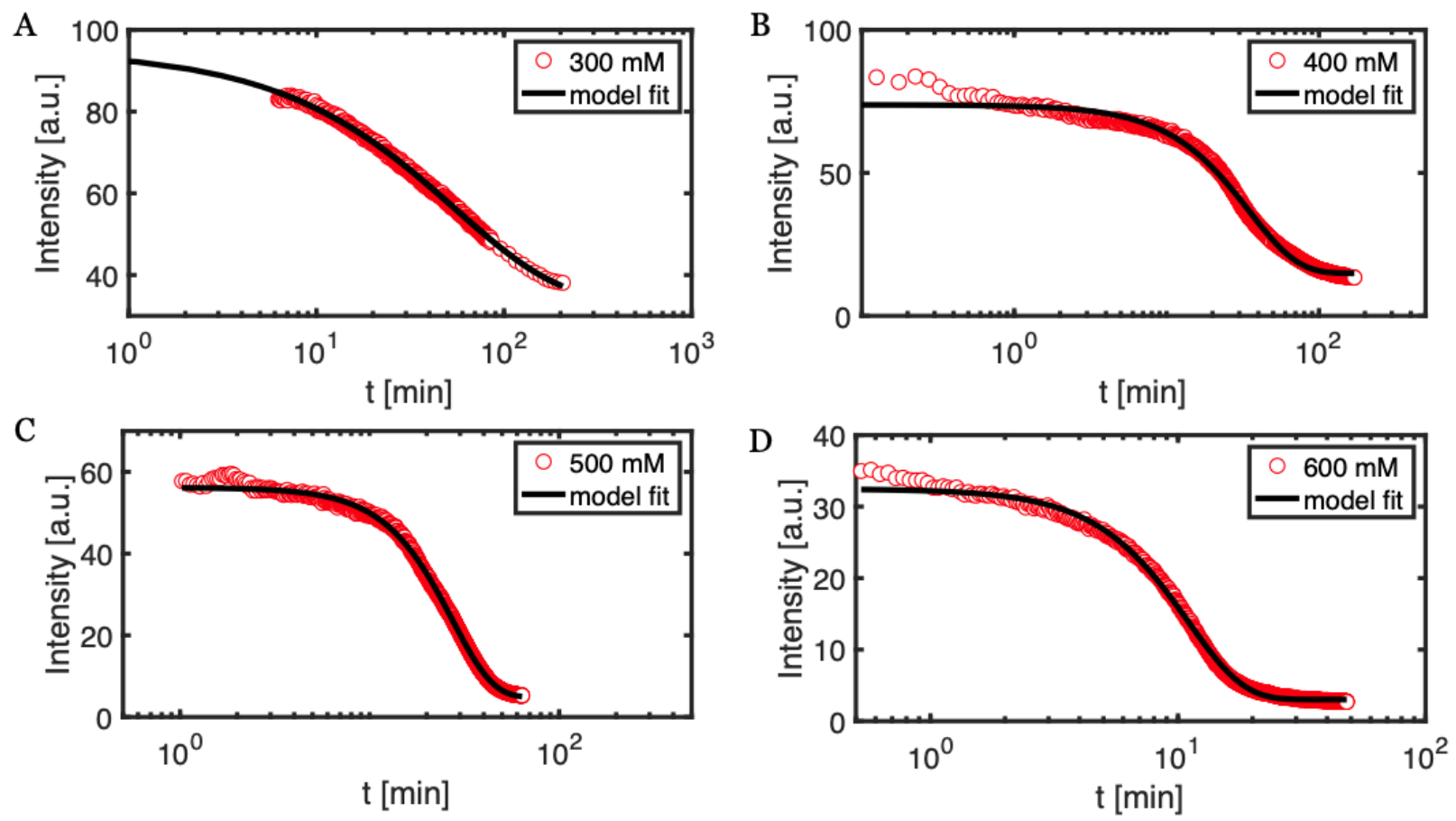

Figure 3: The salt-induced scattering intensity evolution of PEC micelles at four different salt concentrations: (A) $300 \mathrm{mM}$, (B) $400 \mathrm{mM}$, (C) $500 \mathrm{mM}$, and (D) $600 \mathrm{mM}$. Micelles are made of $1.0 \mathrm{mg} \mathrm{mL}-1 \mathrm{PEO}_{225}-b$ - $\mathrm{PVBTMA}_{100} / \mathrm{PAA}_{158}$ solution in aqueous solution at room temperature. The red points are the experimental data and the black lines represent the fits. The relaxation rates from model fits are 51.87, 35.15, 27.37, and $10.88 \mathrm{~min}$ at salt concentrations of $300,400,500$, and $600 \mathrm{mM}$, respectively. 
molecules. This has been previously observed. ${ }^{51}$ Almost all the values of exponent $\beta$ is larger than 1 but smaller than 2. It seems that neither the single chain expulsion/insertion nor the fission-fusion mechanism are solely applicable, especially at high temperatures and low salt concentrations. However, in the following, by applying scaling law of conventional ABC micelles to PEC micelles with consideration of the salt and temperature dependence on the interfacial tension and ionic complex phase density, we demonstrate that the saltinduced dissociation of PEC micelles can be well explained by a micelle fission model which separates the dissociation pathway into three stages. Additionally, the mismatches between experimental data and the model fits at the onset of the dissociation will be explained under the context of the micelle fission model.

Micelle Fission Model. Our model considers the micelle dissociation process in three successive stages, as depicted in Figure 5. First, upon the addition of salt or the temperature increment, the initial micelle undergoes an instantaneous swelling or shrinkage with the changes of the interfacial tension and the complex density of the core, as denoted by $\gamma\left(C_{s}, T\right)$ and $\phi\left(C_{s}, T\right)$ respectively. This step is thought to be fast and cannot entirely be resolved experimentally, although some evidence is presence in the scattering data at initial time points, which will be discussed later. Second, this unstable and short-lived micelle tends to separate into two intermediate micellar aggregates, with the cores in contact and the corona areas overlapping. This is believed to be the rate-limiting step because it involves the compartmentation of the polymer chains in the core and redistribution of the coronaforming chains. Third, the nascent micellar aggregates separate into individual assemblies with the elimination of the corona overlap. The three-staged dissociation model is depicted in Figure 4.

Mathematically, the whole process can be represented as:

$$
M_{\text {init }}\left(P_{0}, \gamma_{0}, \phi_{0}\right) \stackrel{\tau_{1}}{\rightarrow} M_{\text {swell }}\left(P_{0}, \gamma, \phi\right) \stackrel{\tau_{2}}{\rightarrow} M_{\text {frag }}\left(P_{1}, P_{2}, \gamma, \phi\right) \stackrel{\tau_{3}}{\rightarrow} M_{f i n}\left(P_{1}, P_{2}, \gamma, \phi\right)
$$




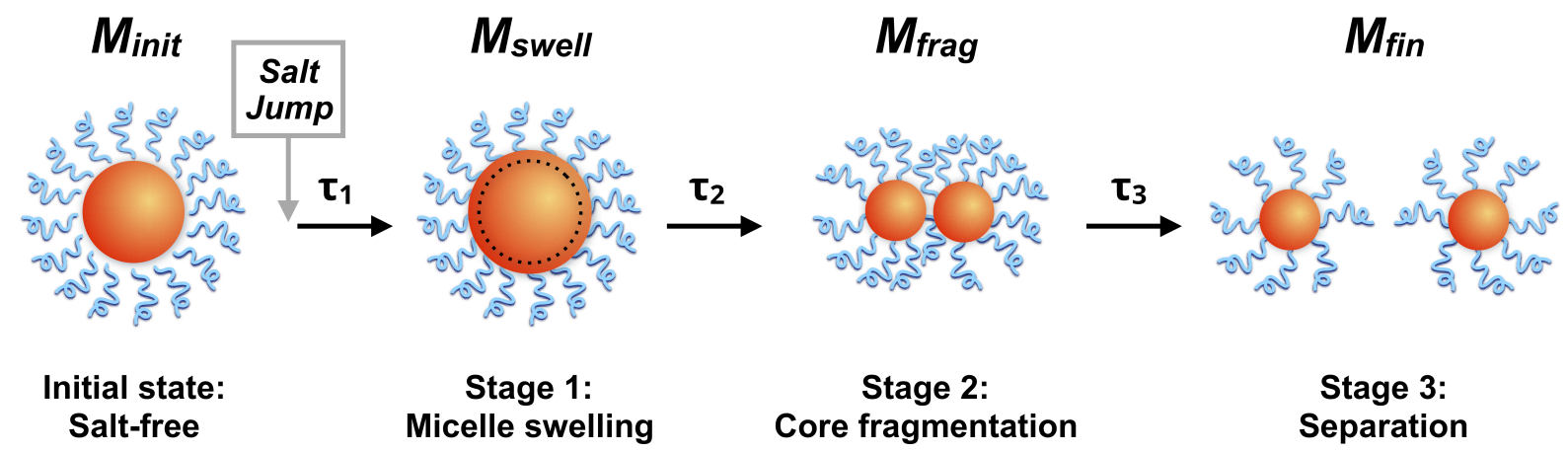

Figure 4: Schematic representation of the three-staged micelle dissociation model.

where $M$ denotes the stage of the micelles, $P_{0}, \gamma_{0}$, and $\phi_{0}$ are the aggregation number, interfacial tension, and core polymer density of the initial micelles, respectively. $P_{1}$ and $P_{2}$ are the aggregation number of the two intermediate micelles $\left(P_{1}+P_{2}=P_{0}\right)$. It is assumed that changes of the interfacial tension and core density complete instantaneously upon the salinity or temperature jump and are invariant afterward, as denoted by the constant values of $\gamma$ and $\phi$. The rate of each step are characterized by $\tau_{1}, \tau_{2}$, and $\tau_{3}$, respectively.

PEC Micelle in Salt-Free Conditions. It is well known that the total free energy of a polymeric micelle in a salt-free solution, $F$, comes from three contributions: (1) $F_{\text {corona }}$ that accounts for the stretching of the coronal blocks in the corona region, (2) $F_{\text {int }}$ that represents the excess free energy of the corona-core interface, and (3) $F_{\text {core }}$ responsible for the extension of the core chains. ${ }^{31,52-54}$ Thus, it can be written as:

$$
\begin{aligned}
F & =F_{\text {corona }}+F_{\text {int }}+F_{\text {core }} \\
& =C_{1} P^{3 / 2}+C_{2}\left(\frac{N_{\text {ionic }} \nu}{\phi}\right)^{2 / 3} P^{2 / 3} \gamma+C_{3}\left(N_{\text {ionic }} \nu\right)^{-1 / 3} \phi^{-2 / 3} P^{5 / 3}
\end{aligned}
$$

where $\nu$ is the mass per charged monomer unit, $P$ is the aggregation number, and $C_{i}$ are coefficients. $C_{1}=\ln [(R+H) / R]$, where $R$ is the size of the core and $H$ is the thickness of the corona. In our case, $C_{1}$ is close to 0.35 , and $C_{2}$ is estimated to be $4 \pi^{1 / 3} 3^{2 / 3}=4.84$. The first and the third terms are of the same order, but usually the third one is ignored in dilute micelle solutions. ${ }^{31,52}$ The minimization of the free energy with respect to $P$ leading to the scaling 
law for PEC micelles in the absence of salt: the equilibrium properties of the PEC micelles are characterized by the aggregation number $P_{0}$, core radius $R_{0}$, core complex density $\phi_{0}$, and core-solvent interfacial tension $\gamma_{0}$. Thus, the equilibrium aggregation number, $P_{e q}$, and core radius, $R_{e q}$, of PEC micelles are given by:

$$
\begin{aligned}
& R_{e q} \simeq \gamma^{2 / 5}\left(N_{\text {ionic }} \nu / \phi\right)^{3 / 5} \\
& P_{e q} \simeq \gamma^{6 / 5}\left(N_{\text {ionic }} \nu / \phi\right)^{4 / 5}
\end{aligned}
$$

Step 1: Micelle Swelling. As mentioned above, in sharp contrast to nonionic ABC micelles, PEC micelles possess a water-rich core where oppositely charged polymers are complexed via ionic interactions. The core-solvent interfacial tension and water fraction are subject to change with the surroundings. The addition of salt leads to the decrease of the Debye screening length, $\kappa^{-1}$, as $\kappa^{-1}=\left(8 \pi l_{B} N_{A} I\right)^{-1 / 2}$, where $l_{B}$ is the Bjerrum length, $N_{A}$ is the Avogadro's constant, and $I$ is the ionic strength expressed in molar (M or mol $\mathrm{L}^{-1}$ ). The salt-induced weakening of the long-range Coulomb interaction between opposite charges results in the swelling of the polyelectrolyte complex phase in micelle cores, which consequently decreases the density of the core and the complex-solvent interfacial tension. This step is assumed to be fast due to the rapid diffusion of salt ions and characterized by a relaxation time $\tau_{1}$. The aggregation number of the swollen micelles remain the same as that of the equilibrium micelles in salt-free solutions, $P=P_{0}$. Thus, the core radius of the swollen micelles, $R$, can be derived following the relationship, $P_{0} N_{\text {ionic }} \nu=(3 / 4) \pi R^{3} \phi=(3 / 4) \pi R_{0}^{3} \phi_{0}$, and written as

$$
R=R_{0}\left(\frac{\phi_{0}}{\phi}\right)^{1 / 3}
$$

Step 2: Micelle Core Fragmentation. Upon the salt jump, the swollen micelles are in a metastable state and tend to evolve into two charge-neutralized aggregates with aggregation numbers $P_{1}$ and $P_{2}$. The nascent aggregates continues to split until a final aggregate number is reached. We characterize the relaxation time for the chain reaction-like process with $\tau_{2, i}$. 
Unimer expulsion pathway is favored when $P_{1}$ or $P_{2}$ equals to 1 . However, in the following, we are going to demonstrate that micelle fission is a more effective pathway to reach new equilibrium states with a significant decrease in the aggregation number. This first split reaction is characterized by the relaxation time $\tau_{2,1}$ and is assumed to be rate-limiting. The activation energy is given by: ${ }^{29,31}$

$$
\Delta F=F\left(P_{1}\right)+F\left(P_{2}\right)-F(P)
$$

The free energy of a micelle can be rewritten as $F(P)=(3 / 2) P_{e q}^{5 / 6} P^{2 / 3}+P^{3 / 2}$, combing Equation (7) and (9). We define $\Omega \equiv P_{1} / P$, denoting the amount of the fissionable polymer chains. Then, the activation energy can be rewritten as:

$$
\Delta F=\frac{3}{2} P_{e q}^{5 / 6} P^{2 / 3}\left[\Omega^{2 / 3}+(1-\Omega)^{2 / 3}-1\right]+P^{3 / 2}\left[\Omega^{3 / 2}+(1-\Omega)^{3 / 2}-1\right]
$$

For $\Omega=1 / 2$, the activation energy becomes negative when $P>2 P_{e q}$, meaning the micelle fission is thermodynamically favorable. This may not stand for some cases in which a small perturbation is induced and the aggregation number does not change markedly; however, it certainly holds true in the irreversible salt-induced micelle dissociation experiments here, because the final size of the micelle after salt jump is very small in most cases $\left(P_{e q}\right.$ is close to zero). In other words, the first term in Equation (12) is negligible, and the second term, which is always negative, reaches its minimum when $\Omega=1 / 2$, meaning fission into two equal-sized aggregates is the most efficient way to decrease the free energy. It should be pointed out that, under this analysis, albeit less efficient, unimer expulsion may also exist.

Further, in order to relate the fission activation energy to the micelle attributes, we rewrite the free energy into

$$
F=C_{1} P^{3 / 2}+4 \pi R^{2} \gamma
$$

Combing Equation (8), (9), and (11), we can get the expression of the fission activation 
energy, which reads

$$
\begin{aligned}
\Delta F & =\pi R^{2} \gamma+C_{1}\left(2^{-1 / 2}-1\right) P^{3 / 2} \\
& =\left[\pi \frac{\gamma_{0}^{4 / 5}}{\phi_{0}^{8 / 15}} \frac{\gamma}{\phi^{2 / 3}}-\frac{3 C_{1}}{10} \frac{\gamma_{0}^{9 / 5}}{\phi_{0}^{6 / 5}}\right] \nu^{6 / 5} N_{\text {ionic }}^{6 / 5}
\end{aligned}
$$

The second term in the bracket in negligible compared to the first term. In turn, the relaxation time of micelle core fragmentation can be estimated as

$$
\tau_{2,1} \simeq \exp (\Delta F)
$$

Thus, the master equation for the micelle fission relaxation time reads

$$
\ln \tau_{2,1} \simeq \pi \frac{\gamma_{0}^{4 / 5}}{\phi_{0}^{8 / 15}} \frac{\gamma}{\phi^{2 / 3}} \nu^{6 / 5} N_{i o n i c}^{6 / 5}
$$

The successive fission reactions proceed in an analogous way to the first fission, i.e., the nascent aggregate splits into two equal-sized smaller aggregates. The interfacial tension coefficient, $\gamma$, and core density, $\phi$, remain the same since the salt concentration is constant in this process. So, the total relaxation time for the micelle fragmentation is given as

$$
\ln \tau_{2}=\left[\sum_{i=2}^{\infty} 2^{-i}+1\right] \pi \frac{\gamma_{0}^{4 / 5}}{\phi_{0}^{8 / 15}} \frac{\gamma}{\phi^{2 / 3}} \nu^{6 / 5} N_{\text {ionic }}^{6 / 5}
$$

From Equation (17), we can see that the further fission steps follow the same scaling law and their relaxation rates are in a geometric sequence with a common ratio of $1 / 2$. If we consider the fission has infinite steps, the total relaxation time for the further steps, $\sum_{i=2}^{\infty}(1 / 2)^{i} \ln \tau_{2,1}$, is estimated to be $\frac{1}{2} \ln \tau_{2,1}$.

Step 3: Micelle Separation. The nascent aggregates from fission are initially in contact, but the coronal chains at the interface tend to stretch into the solvent, which renders the two aggregates drift apart. This process increases the system entropy and the relaxation time, $\tau_{3}$, is considered negligible.

Effect of Salt Concentration. In the following, we aim to investigate the dependencies 
of the relaxation time on salt concentrations, solution temperature, and the length of the charged blocks, and compare our experimental data with theoretical predictions. From Equation (17), the scaling law between the relaxation time and salt concentration reads:

$$
\ln \tau_{2} \approx \frac{\gamma\left(C_{s}\right)}{\phi^{2 / 3}\left(C_{s}\right)}
$$

where $\gamma\left(C_{s}\right)$ and $\phi\left(C_{s}\right)$ represent the dependencies of the interfacial tension and core density on the salt concentration, $C_{s}$, respectively.

The physics of the polymer complexes in micelle cores can be treated as bulk complexes of phase-separated homo-polyelectrolytes, although the space-confinement is missing for the bulk. At stoichiometric conditions, the PEC is a ternary system in which water, polyelectrolytes, and salt coexist. Previous efforts to illustrating the role of salt concentration on interfacial tension have been undertaken from experiments, theories, and simulation. For example, Qin et al. derived an analytical expression based on the Voorn-Overbeek theory for the interfacial tension in near-critical regimes written as $\gamma \propto\left(1-C_{s} / C_{c r}\right)^{3 / 2} .{ }^{55}$ Rumyantsev and coworkers predicted theoretically that, for weakly charged polyelectrolytes, the interfacial tension should follow $\gamma \propto C_{s}^{-2}{ }^{56}$ Yet, a universal expression is still lacking. Here, we adopt the expression reported by Spruijt et al. because of the structural similarities between their polyelectrolytes and ours. ${ }^{57}$ It reads as $\gamma \propto\left(\frac{1}{\sqrt{C_{s}}}-\frac{1}{\sqrt{C_{c r}}}\right)^{3 / 2}$. Details of this expression are shown in their work. Further, the dependence of the complex density on the salt concentration is approximated to be $\phi \propto C_{s}^{-1}$ according to the data reported in experiment and computer simulations by our group. ${ }^{58}$ Thus, the relaxation time scales with salt concentration in a function of

$$
\ln \tau_{2} \propto C_{s}^{2 / 3}\left(\frac{1}{\sqrt{C_{s}}}-\frac{1}{\sqrt{C_{c r}}}\right)^{3 / 2}
$$

Using this relationship, we fit the micelle relaxation rates at four different salt concentrations, and the results are shown in Figure 5. The fit is reasonably good with a residual 


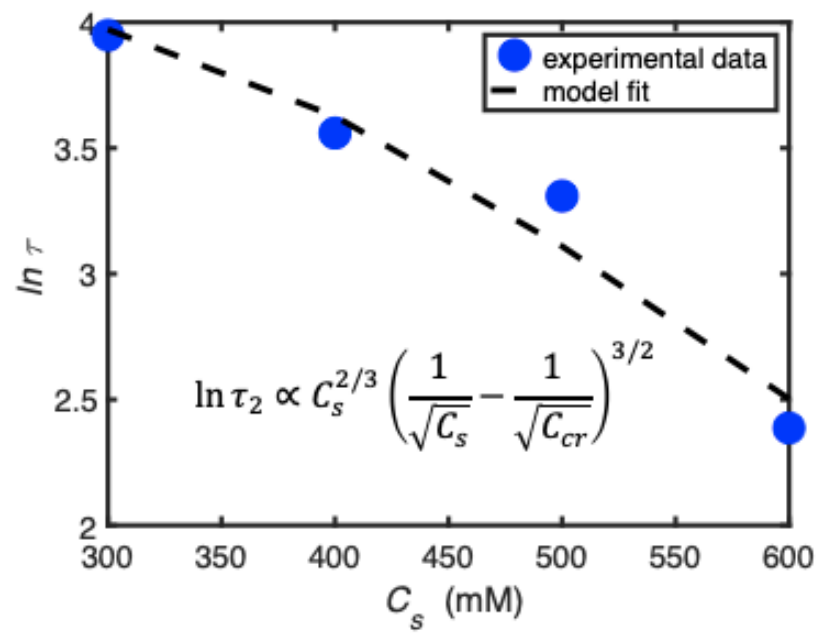

Figure 5: Micelle fission rate as a function of salt concentration. Blue circles corresponding to experimental data obtained from time-dependent light scattering. Black dash lines shows the theoretical predictions from the proposed model.

sum of squares of 0.06 .

Effect of Temperature. Solution temperature has influence on both the core-solvent interfacial tension and the core density. Although the term of temperature is absent in Equation (17), according to the Debye-Huckle theory, the interaction strength of oppositely charged blocks is a function of temperature. ${ }^{59}$ The interaction strength $\alpha$ is defined as $\alpha \equiv$ $\left(16 \pi^{2} / 3\right)\left(l_{B}^{3} / v\right)^{1 / 2} \propto l_{B}^{3 / 2}$, where $l_{B} \equiv e^{2} / 4 \pi \varepsilon_{0} \varepsilon_{r} k_{B} T$ is the Bjerrum length. The interfacial tension scales with the interaction strength following a relationship of $\gamma \propto \alpha^{1 / 2} \propto l_{B}^{3 / 4} \propto$ $T^{-3 / 4}$. The core density is viewed as constant in the temperature range studied here, which is supported by the evidence that the micelles remained the same size over the temperature range. Thus, we can derive the temperature dependence of micelle fission rate as $\ln \tau_{2} \propto$ $T^{-3 / 4}$. The expression fits the experimental data well with a squared residual of 0.01 , as shown in Figure 6.

Effect of Charged Block Length. From Equation (17), the dependence of the relaxation time on the length of ionic blocks reads

$$
\ln \tau_{2} \propto N_{i o n i c}^{6 / 5}
$$




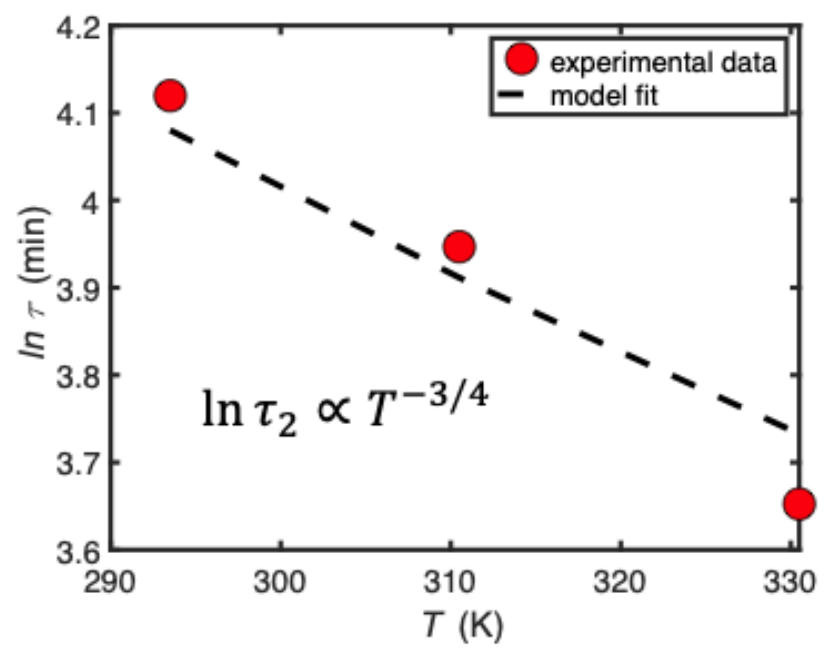

Figure 6: Micelle fission rate as a function of temperature. Red circles correspond to the experimental data from the time-dependent light scattering experiments. Black dashes shows the theoretical prediction.

The dependence of the micelle fission rate on ionic block length is drawn in Figure 7 . It is shown that the logarithmic fission rate increases with the degree of polymerization of the charged block with a power law of $6 / 5$. Although we do not have experimental data to check this prediction so far, this relationship can be experimentally examined in the future and also providing guidance for designing carriers with customized lifetime.

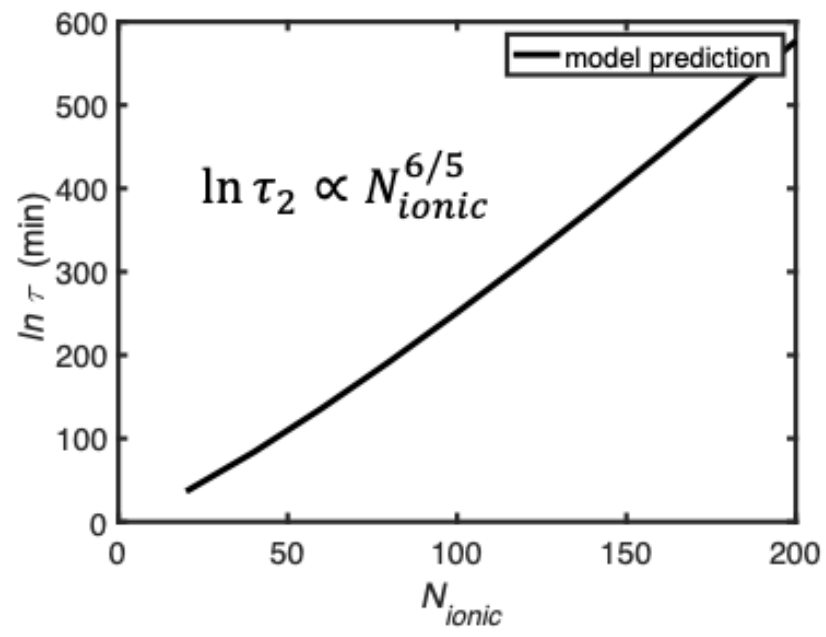

Figure 7: Theoretical prediction of the micelle fission rate as a function of ionic block length.

As mentioned previously, we see mismatches between the experimental data and the quantitative model at the beginning of the dissociation process. This phenomenon is more 
obvious in the salt-dependence case than in the temperature dependence case. We speculate that is where the micelle swelling or shrinking happens - the fast first step characterized by a relaxation rate $\tau_{1}$. According to Equation (4), the model assumes the scattering intensity is expressed as a function of the time-dependent micellar aggregation number. For a given aggregation number, scattering density scales with micelle sizes as $I \propto R_{\text {micelle }}^{3}$ During step one, the average aggregation number remains the same, but micelle swells, albeit fast, which results in the differences between the experimental data and model fits. This explains why the mismatch in the salt-dependence case is more evident than that in the temperaturedependence case since the micelle size barely changes with temperature increase. Decoupling the contributions from micelle swelling is experimentally difficult, if not impossible. During micelle core fragmentation, both the micelle size and aggregation number decrease, which leads to the precipitous drop of the scattering intensity. In the last step, where nascent aggregates drift apart, scattering intensities remain the same because neither the micelle size nor the aggregation number changes. The discussion also excludes the micelle separatin step that is characterized by a rate of $\tau_{3}$ because of the absence of coronal contribution as mentioned in the previous context.

\section{CONCLUSIONS}

In summary, the mechanism of dissociation kinetics in polyelectrolyte complex micelles is demonstrated by a combination of light scattering experiments and theoretical modeling. The theoretical framework for PEC micelle dissociation is built upon scaling law of PEC micelles, kinetic theories for amphiphilic block copolymers, and theories on polyelectrolyte complexes. Salt-induced micelle disassembly is characterized as a three-staged process. First, upon salt addition micelles immediately swell because of the weakening of the electrostatic interaction. Second, micelles undergo a successive chain rearrangement where micelle cores tend to fragment into two charge-balanced aggregates. Theoretical predictions show that 
splitting into two equal-sized aggregates is the most efficient way to reach new equilibrium states, which leads to the sharp drop of the scattering intensity, as we observe in the timedependent light scattering experiments. Third, the nascent aggregates separate from each other.

Moreover, we illustrate the dependencies of micelle fission relaxation rates on salt concentration, temperature, and charged block length. By taking into account the recent advances in understanding the relationship between interfacial tension and salt concentration/temperature in polyelectrolyte complexes, we derive an analytical expression for fission relaxation rates. The comparison of the predictions with our experimental data is in good agreement. We also make a prediction on the scaling law between the relaxation rate and the

ionic block as $\ln \tau \propto N_{\text {ionic }}^{6 / 5}$, which can be further experimentally examined. We anticipate that these findings will provide insights on the fundamental understanding of micelle dissociation and encourage future effort on the kinetics of polyelectrolyte-based self-assemblies.

\section{EXPERIMENTAL}

Polymer Synthesis. PEO- $b$-PVBTMA was synthesized by aqueous reversible additionfragmentation chain transfer (RAFT) polymerization. The details have been previously reported by us previously. ${ }^{35}$ The poly(acrylic acid sodium salt) $\left(\mathrm{PAA}_{158}, M_{w}=14,800\right.$ g mol-1) was purchased from Polymer Source Inc. with confirmed molecular weight and chemical structures.

Micelle Preparation. Micelle preparation and characterization were performed according to the protocol established in our previous work. ${ }^{36}$ Briefly, polymer stock solutions were prepared by dissolving polyelectrolytes in Milli-Q water at the total concentration of $5 \mathrm{mg}$ $\mathrm{mL}^{-1}$ and filtering solutions through PVDF filters (average diameter $\approx 220 \mathrm{~nm}$ ). Micellar assemblies were formed by directly mixing the polycation and polyanion solutions under stoichiometric conditions. The order of addition was checked and did not affect the assembly 
features. All the samples were set aside for at least $24 \mathrm{~h}$ before any experiments.

Dynamic Light Scattering. Dynamic light scattering was carried out on a Brookhaven Instruments BI-200SM Research Goniometer System with a $637 \mathrm{~nm}$ incident laser. The scattering vector, $q$, is defined by $q=\frac{4 \pi n}{\lambda} \sin \left(\frac{\theta}{2}\right)$, where $\lambda$ is the wavelength of the incident light, $n$ is the refractive index of the solution, and $\theta$ is the angle between the incident and scattering laser. The fluctuation of the electric field generated by the ensemble collection of the solution particles under Brownian diffusion can be expressed using a field autocorrelation function: $\int_{0}^{\infty} E(t) E(t+\tau) d \tau=e x p^{-D q^{2} \tau}$, where $E$ is the electric field, $\tau$ is the delay time, and $D$ is the diffusion coefficient. The experimentally measured intensity correlation function, $G_{2}$, is equivalent to the actual electric field autocorrelation, $G_{1}$, through the Seigert relationship: $G_{2}(\tau)=1+\gamma G_{1}(\tau)$, where $\gamma$ is a coherence factor indicating the efficiency of the photo collection system.

The hydrodynamic radii of scatterers under Brownian diffusion was calculated via StokesEinstein relationship: $R_{h}=\left(k_{B} T\right) / 6 \pi \eta D$, where $k_{B}$ is the Boltzmann constant, $T$ is the absolute temperature, and $\eta$ is the viscosity of the solution, here as taken that of water at the investigated temperature. Diffusion coefficient and polydispersity index were extracted by fitting intensity correlation functions to a second order-expanded single exponential as below: $G_{2}=A\left[1+B \exp \left(-2 \Gamma \tau+\mu_{2} \tau^{2}\right)\right]$, where $A$ is the amplitude or intercept of the correlation function, $B$ is the baseline, $\Gamma$ is the decay rate, and $\mu_{2}$ is proportional to the distribution width. The polydispersity index is expressed as: $P d I=\mu_{2} / 2 \Gamma=\sigma^{2} /\left(2 R_{h}^{2}\right)$, where $\sigma$ is the standard deviation of a hypothetical Gaussian distribution centered on $R_{h}$. The size distribution was obtained using REPES algorithm.

Multi-angle measurement was conducted using a script that written by the $\mathrm{Cmm}$ scripting language. Correlation functions were typically collected at 12 scattering angles from $30^{\circ}$ to $140^{\circ}$ at $10^{\circ}$ increments, and running duration of each angle was at least 1 minute. The angular dependence of diffusion coefficient was acquired by plotting $q^{2}$ versus decay rate $\Gamma$. A linearity over a range of scattering angles is a good indication of isotropic scatters, i.e., 
spheres when the radius is larger than $\lambda / 20 \mathrm{~nm}$. The correlation function at each angle was fitted to a first-order single exponential relaxation function in MATLAB.

Time-Resolved Static Light Scattering. The time-resolved scattering experiments were carried out on a Brookhaven Instruments BI-200SM Research Goniometer System with a $637 \mathrm{~nm}$ incident laser. A $\mathrm{cmm}$ script is written to control the time-resolved measurements automatically, which is deadtime about 1 second. The acquisition time (usually 30 seconds) and a readout pause between two acquisitions are optimized according to different circumstance. All experiments were conducted at the angle of $90^{\circ}$.

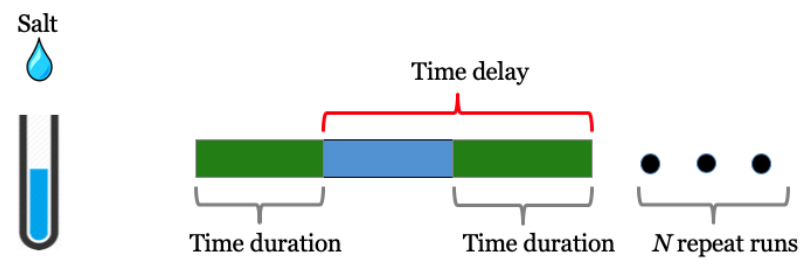

Scheme 1: Schematic representation of the experimental setup of time-resolved static light scattering experiments.

Small-Angle X-Ray Scattering. All SAXS experiments were carried out on the Biological Small Angle Scattering Beam Line BL4-2 at the Stanford Synchrotron Radiation Lighthouse (SSRL), SLAC National Accelerator Laboratory. The sample-to-detector distance was set to $3.5 \mathrm{~m}$ and X-rays with the wavelength of $\lambda=1.38 \mathrm{~A}(9 \mathrm{keV})$ were utilized for the measurements. Using a Pilatus3 X 1M detector (Dectris Ltd, Switzerland) the setup covered a range of momentum transfer $q \approx 0.0025-0.17 \mathrm{~A}^{-1}$ where $q$ is the magnitude of the scattering vector. Aliquots of $30 \mu \mathrm{L}$ of the polyelectrolyte micellar suspension were loaded onto the automated fluid sample loader at the beamline. Series of sixteen consecutive one second exposures were collected first from the buffer blank (pure water) and followed by the micelle samples of different concentrations. Solutions were oscillated in a stationary quartz capillary cell during data collection to maximize the exposed sample volume and reduce the radiation dose per unit volume of sample. The collected data were radially integrated, analyzed for radiation damage, and buffer subtracted using the automated data reduction 
pipeline at the beam line. Only data that did not show any signs of the effects of radiation damage were included in the final average for each sample concentration. More details on the SAXS data collection are available in the literature. ${ }^{60}$ The X-ray scattering length densities of the polymers are calculated using the SLD calculator provided by NIST and are shown in Table 2.

Table 2: The calculated scattering length densities of polymers and water.

\begin{tabular}{llcc}
\hline Chemical & Formula & Density $\left(\mathrm{g} \mathrm{cm}^{-3}\right)$ & SLD $\left(10^{-6} \AA^{-1}\right)$ \\
\hline Water & $\mathrm{H}_{2} \mathrm{O}$ & 1.00 & 9.47 \\
PEO & $\mathrm{CH}_{3}\left(\mathrm{C}_{2} \mathrm{H}_{4} \mathrm{O}\right)_{\mathrm{n}}$ & 1.13 & 10.50 \\
PAA & $\left(\mathrm{C}_{3} \mathrm{H}_{3} \mathrm{O}_{2} \mathrm{Na}\right)_{\mathrm{n}}$ & 1.50 & 13.04 \\
PVBTMA & $\left(\mathrm{C}_{12} \mathrm{H}_{18} \mathrm{NCl}\right)_{\mathrm{n}}$ & 1.20 & 12.00 \\
\hline
\end{tabular}

Micelle samples were fitted using a model that contains polydisperse core-shell sphere and a polydisperse Gaussian coil function, which has been reported by us previously. ${ }^{34}$ The details of the model can be found in the Supporting Information.

Cryogenic Electron Microscopy. FEI Tecnai ${ }^{\mathrm{TM}}$ G2 Spirit BioTWIN TEM was used to image samples set at an accelerating voltage of $120 \mathrm{kV}$ onto a LaB6 emitter at -178 ${ }^{\circ} \mathrm{C}$. Samples vitrification was done on a FEI vitrobot system coupled with a Bal-Tec highpressure freezer. A droplet of about $3.0 \mu \mathrm{L}$ samples containing polyelectrolyte complex assemblies was pipetted onto a carbon/Formvar grid that is cleaned by a PELCO easiGlow glow discharge instrument. The grid was blotted for 3 seconds and was quenched rapidly in liquid ethane, and subsequently transferred to a single-tilt cryo holder for visualization on a Cryo-EM dedicated FEI Talos 2000kV FEG electron microscopy.

\section{Acknowledgement}

The authors gratefully thank the financial support from the U.S. Department of Commerce, National Institute of Standards and Technology (NIST), through the Center for Hierarchical Materials Design (CHiMaD). J.M.T. acknowledges support from the NIST- CHiMaD 
Postdoctoral Fellowship. The authors gratefully thank Artem M. Rumyantsev, PhD, for his insightful discussions. The authors thank Tera Lavoie, PhD, in the Advanced Electron Microscopy Facility at the University of Chicago for her expertise and contributions to the cryo-imaging. The authors also thank Thomas M. Weiss, PhD, Ivan Rajkovic, PhD and Tsutomu Matsui, PhD, at the Stanford National Accelerator Laboratory for their assistance in scattering experiments and insightful discussions. Use of the Stanford Synchrotron Radiation Lightsource, SLAC National Accelerator Laboratory, is supported by the U.S. Department of Energy, Office of Science, Office of Basic Energy Sciences under Contract No. DE-AC0276SF00515. The SSRL Structural Molecular Biology Program is supported by the DOE Office of Biological and Environmental Research, and by the National Institutes of Health,

National Institute of General Medical Sciences (including P41GM103393). The contents of this publication are solely the responsibility of the authors and do not necessarily represent the official views of NIGMS or NIH.

\section{References}

(1) Brangwynne, C. P.; Tompa, P.; Pappu, R. V. Nat. Phys. 2015, 11, 899-904.

(2) Perutz, M. Science 1978, 201, 1187-1191.

(3) Sheinerman, F. B.; Norel, R.; Honig, B. Pediatrics 2000, 10, 153-159.

(4) Walther, T. H.; Ulrich, A. S. Curr. Opin. Struct. Biol. 2014, 27, 63-68.

(5) Lueckheide, M.; Vieregg, R.; Bologna, A. J.; Leon, L.; Tirrell, M. V. Nano Lett. 2018, $7111-7117$.

(6) Vieregg, J. R.; Lueckheide, M.; Marciel, A. B.; Leon, L.; Bologna, A. J.; Rivera, J. R.; Tirrell, M. V. J. Am. Chem. Soc. 2018, 140, 1632-1638.

(7) Kanasty, R.; Dorkin, J. R.; Vegas, A.; Anderson, D. Nat. Mater. 2013, 12, 967-977. 
(8) Priftis, D.; Laugel, N.; Tirrell, M. Langmuir 2012, 28, 15947-15957.

(9) Priftis, D.; Tirrell, M. Soft Matter 2012, 8, 9396-9405.

(10) Priftis, D.; Xia, X.; Margossian, K. O.; Perry, S. L.; Leon, L.; Qin, J.; De Pablo, J. J.; Tirrell, M. Macromolecules 2014, 47, 3076-3085.

(11) Priftis, D.; Leon, L.; Song, Z.; Perry, S. L.; Margossian, K. O.; Tropnikova, A.; Cheng, J.; Tirrell, M. Angew. Chemie Int. Ed. 2015, 54, 11128-11132.

(12) Perry, S. L.; Leon, L.; Hoffmann, K. Q.; Kade, M. J.; Priftis, D.; Black, K. A.; Wong, D.; Klein, R. A.; Pierceiii, C. F.; Margossian, K. O.; Whitmer, J. K.; Qin, J.; Pablo, J. J. D.; Tirrell, M. Nat. Commun. 2015, 6, 6052.

(13) Marciel, A. B.; Chung, E. J.; Brettmann, B. K.; Leon, L. Adv. Colloid Interface Sci. 2017, 239, 187-198.

(14) Marciel, A. B.; Srivastava, S.; Tirrell, M. V. Soft Matter 2018, 18-22.

(15) de Vries, R.; Cohen Stuart, M. Curr. Opin. Colloid Interface Sci. 2006, 11, 295-301.

(16) Zhao, Q.; Lee, D. W.; Ahn, B. K.; Seo, S.; Kaufman, Y.; Israelachvili, J. N.; Waite, J. H. Nat. Mater. 2016, 15, 407-412.

(17) Black, K. A.; Priftis, D.; Perry, S. L.; Yip, J.; Byun, W. Y.; Tirrell, M. ACS Macro Lett. 2014, 3, 1088-1091.

(18) McCall, P. M.; Srivastava, S.; Perry, S. L.; Kovar, D. R.; Gardel, M. L.; Tirrell, M. V. Biophys. J. 2018, 114, 1636-1645.

(19) Kataoka, K.; Harada, A.; Nagasaki, Y. Adv. Drug Deliv. Rev. 2012, 64, 37-48.

(20) Cabral, H.; Miyata, K.; Osada, K.; Kataoka, K. Chem. Rev. 2018, 118, 6844-6892. 
(21) Hunt, J. N.; Feldman, K. E.; Lynd, N. a.; Deek, J.; Campos, L. M.; Spruell, J. M.; Hernandez, B. M.; Kramer, E. J.; Hawker, C. J. Adv. Mater. 2011, 23, 2327-2331.

(22) Srivastava, S.; Andreev, M.; Levi, A. E.; Goldfeld, D. J.; Mao, J.; Heller, W. T.; Prabhu, V. M.; De Pablo, J. J.; Tirrell, M. V. Nat. Commun. 2017, 8, 14131.

(23) Lemmers, M.; Spruijt, E.; Akerboom, S.; Voets, I. K.; Aelst, A. C. V.; Stuart, M. A. C.; Gucht, J. V. D. Langmuir 2012, 28, 12311-123118.

(24) Kabanov, A. V.; Kabanov, V. A. Adv. Drug Deliv. Rev. 1998, 30, 49-60.

(25) Gwan, T.; Hoon, J.; Wan, S. Adv. Drug Deliv. Rev. 2006, 58, 467-486.

(26) Mundra, V.; Mahato, R. I. Front. Chem. Sci. Eng. 2014, 8, 387-404.

(27) Voets, I. K.; Keizer, A. D.; Stuart, M. A. C. Adv. Colloid Interface Sci. 2009, 147-148, $300-318$.

(28) Aniansson, E. A. G.; Wall, S. N. J. Phys. Chem. 1975, 79, 857-858.

(29) Halperin, A.; Alexander, S. Macromolecules 1989, 22, 2403-2412.

(30) Esselink, F. J.; Dormidontova, E.; Hadziioannou, G. Macromolecules 1998, 31, 29252932.

(31) Dormidontova, E. E. Macromolecules 1999, 32, 7630-7644.

(32) Burke, S. E.; Eisenberg, A. Langmuir 2001, 17, 6705-6714.

(33) Rharbi, Y. Macromolecules 2012, 45, 9823-9826.

(34) Wu, H.; Ting, J.; Weiss, T.; Tirrell, M. ChemRxiv 2019, Preprint.

(35) Ting, J. M.; Wu, H.; Herzog-Arbeitman, A.; Srivastava, S.; Tirrell, M. V. ACS Macro Lett. 2018, 7, 726-733. 
(36) Wu, H.; Ting, J. M.; Werba, O.; Meng, S.; Tirrell, M. V. J. Chem. Phys. 2018, 149 , 163330.

(37) Haladjova, E.; Mountrichas, G.; Pispas, S.; Rangelov, S. J. Phys. Chem. B 2016, 120, $2586-2595$.

(38) Marras, A. E.; Vieregg, J. R.; Ting, J. M.; Rubien, J. D.; Tirrell, M. V. Polymers 2019, $11,83$.

(39) Pedersen, J. S. 2001, 2839.

(40) Pedersen, J. S.; Svaneborg, C. Curr. Opin. Colloid Interface Sci. 2002, 7, 158-166.

(41) Pedersen, J. S.; Svaneborg, C.; Almdal, K.; Hamley, I. W.; Young, R. N. 2003, I, $416-433$.

(42) Lemmers, M.; Voets, I. K.; Cohen Stuart, M. A.; der Gucht, J. V. Soft Matter 2011, 7, 1378.

(43) Avrami, M. J. Chem. Phys. 1940, 8, 212-224.

(44) Ruegg, M. L.; Patel, A. J.; Narayanan, S.; Sandy, A. R.; Mochrie, S. G.; Watanabe, H.; Balsara, N. P. Macromolecules 2006, 39, 8822-8831.

(45) Cipelletti, L.; Manley, S.; Ball, R. C.; Weitz, D. A. Phys. Rev. Lett. 2000, 84, 22752278.

(46) Abraham, T.; Giasson, S.; Gohy, J. F.; Jérôme, R.; Müller, B.; Stamm, M. Macromolecules 2000, 33, 6051-6059.

(47) Mihut, A. M.; Chiche, A.; Drechsler, M.; Schmalz, H.; Di Cola, E.; Krausch, G.; Ballauff, M. Soft Matter 2009, 5, 208-213.

(48) Loo, Y. L.; Register, R. A.; Ryan, A. J.; Dee, G. T. Macromolecules 2001, 34, 89688977. 
(49) Xu, J. T.; Fairclough, J. P. A.; Mai, S. M.; Ryan, A. J.; Chaibundit, C. Macromolecules 2002, 35, 6937-6945.

(50) Lund, R.; Willner, L.; Richter, D.; Dormidontova, E. E. Macromolecules 2006, 39, 4566-4575.

(51) Meli, L.; Santiago, J. M.; Lodge, T. P. Macromolecules 2010, 43, 2018-2027.

(52) Birshtein, T. M.; Zhulina, E. B. Polymer 1989, 30, 170-177.

(53) Halperin, A. Macromolecules 1987, 20, 2943-2946.

(54) Rumyantsev, A. M.; Zhulina, E. B.; Borisov, O. V. ACS Macro Lett. 2018, 7, 811-816.

(55) Qin, J.; Priftis, D.; Farina, R.; Perry, S. L.; Leon, L.; Whitmer, J.; Hoffmann, K.; Tirrell, M.; De Pablo, J. J. ACS Macro Lett. 2014, 3, 565-568.

(56) Rumyantsev, A. M.; Zhulina, E. B.; Borisov, O. V. Macromolecules 2018, 51, 37883801.

(57) Spruijt, E.; Sprakel, J.; Cohen Stuart, M. A.; Van Der Gucht, J. Soft Matter 2009, 6, 172-178.

(58) Li, L.; Srivastava, S.; Andreev, M.; Marciel, A. B.; de Pablo, J. J.; Tirrell, M. V. Macromolecules 2018, 51, 2988-2995.

(59) Debye, P.; Huckel, P. Physikalische Zeitschrift. 1923, 24, 185-206.

(60) Smolsky, I. L.; Liu, P.; Niebuhr, M.; Ito, K.; Weiss, T. M.; Tsuruta, H. J. Appl. Crystallogr. 2007, 40, 453-458. 


\section{Graphical TOC Entry}

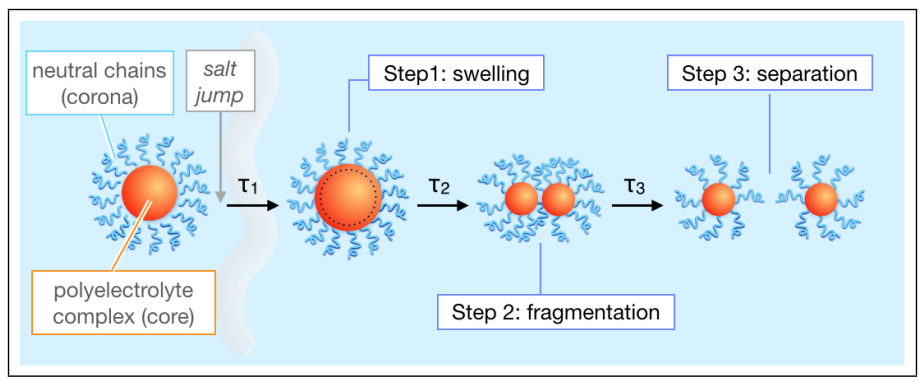

